\title{
Phenylboronic Acid-Modified Polyamidoamine Mediated the Transfection of Polo-Like Kinase-I siRNA to Achieve an Anti-Tumor Efficacy
}

\author{
Gu Gong ${ }^{1, *}$ \\ Xiuhui Tang ${ }^{2, *}$ \\ Jiayuan Zhang ${ }^{2}$ \\ Xiao Liang ${ }^{2}$ \\ Jiebing Yang ${ }^{2}$ \\ Quanshun Li (iD) ${ }^{2}$ \\ 'Department of Orthopaedic Surgery, \\ China-Japan Union Hospital, Jilin \\ University, Changchun, I3003I, People's \\ Republic of China; ${ }^{2}$ Key Laboratory for \\ Molecular Enzymology and Engineering of \\ Ministry of Education, School of Life \\ Sciences, Jilin University, Changchun, \\ 1300I2, People's Republic of China
}

*These authors contributed equally to this work
Correspondence: Quanshun Li; Jiebing

Yang

$\mathrm{Tel} / \mathrm{Fax}+86-43 \mathrm{I}-85 \mathrm{I} 55200$

Email quanshun@jlu.edu.cn;

yangjiebing@Intu.edu.cn
Background: The construction of tumor-targeting carriers with favorable transfection efficiency was of great significance to achieve the tumor gene therapy. The phenylboronic acid-modified polyamidoamine (namely PP) was employed as a carrier for the delivery of Polo-like kinase-1 siRNA (siPlk-1), inducing an obvious anti-tumor response.

Materials and Methods: The interaction between PP and siPlk-1 was evaluated by gel retardation assay. The transfection efficiency and tumor-targeting ability were analyzed by flow cytometry and confocal laser scanning microscopy, using hepatocarcinoma cell line HepG2 as a model. The anti-proliferation effect of PP/siPlk-1 and related mechanism were studied using the strategies of 3-(4,5-dimethylthiazol-2-yl)-2,5-diphenyltetrazolium bromide (MTT) assay, cell apoptosis and cell cycle arrest. The anti-migration effect induced by PP/ siPlk-1 delivery was assayed by wound healing and Transwell migration techniques. Finally, quantitative real-time PCR and Western blotting were performed to measure the expression level of Plk-1 and other key targets.

Results: The derivative PP could achieve the condensation of siPlk-1 into stable nanoparticles at nitrogen/phosphate groups ratio (N/P ratio) of $>3.0$, and it could facilitate the transfection of siPk-1 in a phenylboronic acid-dependent manner. The PP/siPlk-1 nanoparticles exhibited obvious anti-proliferation effect owing to the gene silence of Plk-1, which was identified to be associated with the cell apoptosis and cell cycle arrest at G2 phase. Meanwhile, PP/siPlk-1 transfection could efficiently suppress the migration and invasion of tumor cells.

Conclusion: The derivative PP has been demonstrated to be an ideal tumor-targeting carrier for the delivery of Plk-1 siRNA, exhibiting great potential in the gene therapy of malignant tumors. Keywords: phenylboronic acid, polyamidoamine, tumor-targeting ability, siPlk-1, gene therapy

\section{Introduction}

The rapid development of malignant tumors will lead to millions of death worldwide every year, and cancer has become one of the main causes of human mortality and represents an increasingly serious health problem. ${ }^{1}$ Hepatocellular carcinoma (HCC) is the fifth most common cancer in the world, which accounts for the second leading cause of death among the patients bearing cancers. ${ }^{2}$ Though the strategy breakthroughs in recent years regarding chemotherapy, radiotherapy, immunotherapy and liver transplantation, the treatment outcomes of HCC are still unsatisfactory. ${ }^{3,4}$ Thus, safe and effective techniques are urgently needed to prolong the survival of patients suffering from liver cancers. 
Gene therapy which could efficiently improve the expression level of therapeutic genes or suppress the expression of oncogenes, has been considered as a promising tool in the tumor treatment. ${ }^{5}$ In the gene therapy, RNA interference holds enormous proficiency in restraining the expression of target genes through small interfering RNA (siRNA), and thus it possesses great potential in the treatment of a variety of diseases including cancers. ${ }^{6,7}$ To date, the occurrence and development of malignant tumors has been illustrated to be highly associated with the abnormal expression or dysregulation of cancer-related genes. ${ }^{8}$ For example, Polo-like kinase-1 (Plk-1) plays a crucial role in promoting the progression of cell cycle by participating in the cell division. ${ }^{9}$ Meanwhile, Plk-1 was identified to be overexpressed in many cancers and related to the tumor proliferation and migration. ${ }^{10-12}$ Thus, Plk-1 could be used as a potential candidate for the development of anticancer drugs, and down-regulating the Plk-1 expression has been accepted as an effective route to achieve the tumor gene therapy. ${ }^{13}$

For the use of siRNA as therapeutic drugs, there are many obstacles to be overcome, including the degradation by endogenous RNases, immunogenicity, short half-life, and limited cellular uptake. ${ }^{14,15}$ To achieve the transfection of Plk-1 siRNA (siPlk-1) in tumor cells, various carriers have been successfully developed including atelocollagen, liposomes, cationic polymers and exosome. ${ }^{11,16-23}$ Compared to these carriers, amine-terminated dendrimer polyamidoamine (PAMAM) could condense siRNA to form stable nanoparticles via electrostatic interaction more efficiently, showing favorable transfection efficiency. ${ }^{24-27}$ In addition to prolonging the circulation time of siRNA, PAMAM could protect siRNA from the recognition by immune system. ${ }^{28}$ When the nanoparticles reach tumor sites, the positively charged PAMAM could promote the cellular uptake of siRNA and the release of siRNA from endosomes into cytoplasm via "proton sponges" effect. ${ }^{29}$ In spite of high transfection efficiency, PAMAM lacks the ability to target specific cells, and thus it could not fulfill the requirements in gene therapy. Up to now, there were a number of modification strategies which have been reported to improve the transfection efficiency of PAMAM, as well as the specificity toward tumor cells. ${ }^{30-34}$ In our previous reports, phenylboronic acid (PBA) was conjugated on the surface of PAMAM to construct two types of tumor-targeted derivatives which were employed as carriers to facilitate the delivery of oligonucleotides including miR-34a, DNAzyme and short GC rich
DNA. ${ }^{33-35}$ PBA has been considered as an effective ligand which specifically recognizes the sialic acids overexpressed in various tumor cells. ${ }^{36,37}$ After the modification with PBA, the derivatives could acquire the tumor-targeted ability and achieve favorable gene transfection via the sialic acid-dependent endocytosis pathway. ${ }^{38}$ Thus, we inferred that the PBA-functionalized derivatives could be potentially used as carriers for siPlk-1 delivery and then obtain ideal anti-tumor efficacy.

Herein, a PBA-functionalized polyamidoamine (namely PP) was developed and employed as a carrier to achieve the delivery of siPlk-1, as shown in Scheme 1. After the successful transfection of PP/siPlk-1 nanoparticles, the inhibition of cell proliferation and migration was systematically investigated using human HCC cell line HepG2 as a model.

\section{Materials and Methods Materials}

Amine-terminated G5 PAMAM dendrimer was obtained from Chenyuan Co. (Weihai, China) with the generation of 5.0 and the molecular weight of $28,860 \mathrm{~g} / \mathrm{mol}$. The derivative PP (with PBA degree on the surface of carrier of 7.2) was synthesized by the modification of 4-carboxyphenylboronic acid on PAMAM according to our previous report. ${ }^{33}$ Dulbecco's modified Eagle's medium (DMEM) and fetal bovine serum (FBS) were obtained from Gibco (Grand Island, NY) and Kangyuan Co. (Beijing, China), respectively. TRNzol Universal reagent was acquired from TIANGEN (Beijing, China). Primary antibodies against Plk-1, procaspase 3, procaspase 8, procaspase 9, Bcl-2, p53, poly-ADP-ribose polymerase (PARP) and $\beta$-actin, and horseradish peroxidase (HRP)conjugated secondary antibody were purchased from Abcam (Shanghai, China). The following kits were employed in the present study: Annexin V-FITC/PI apoptosis detection kit, Vazyme Biotech. (Nanjing, China); mitochondrial membrane potential assay kit, Beyotime Biotech. (Jiangsu, China); bicinchoninic acid (BCA) protein assay kit, BioTeke (Beijing, China); PrimeScript RT reagent kit and SYBR Premix Ex Taq kit, TAKARA (Dalian, China). The siPlk-1, negative control (NC), and the carboxyfluorescein (FAM)- or Cyanine5.5 (Cy5.5)labeled forms were synthesized in GenePharma Co. (Suzhou, China) as follow:

siPlk-1: sense: 5'-GCAACCUGCAGUGUAAUAATT-3'; antisense: 5'-UUAUUACACUGCAGGUUGCTT-3'; 


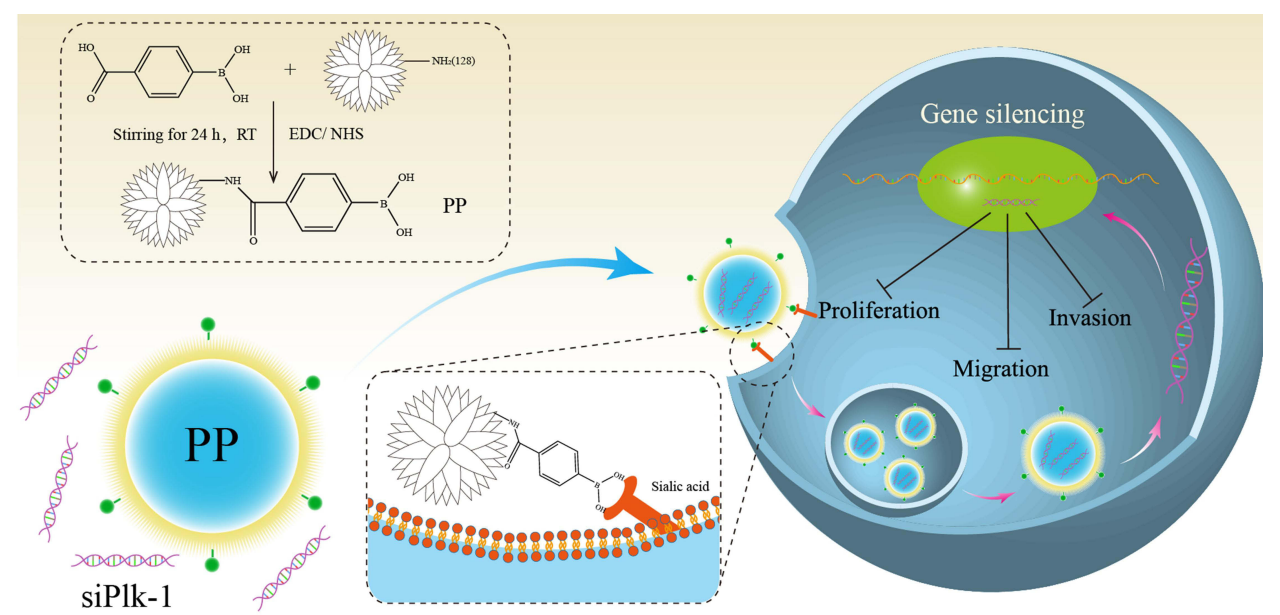

Scheme I Construction of PP derivative and transfection of PP/siPlk-I nanoparticles.

NC: sense: 5'-UUCUCCGAACGUGUCACGUTT-3'; antisense: 5'-ACGUGACACGUUCGGAGAATT-3'.

\section{Preparation and Characterization of PP/ siPlk-I Nanoparticles}

Briefly, PP was gently mixed with siPlk-1 at different N/P ratios (1.0-5.0), and the mixture was incubated at $25{ }^{\circ} \mathrm{C}$ for $30 \mathrm{~min}$ to prepare PP/siPlk-1 nanoparticles. The nanoparticles were then analyzed using $1 \%$ agarose gel electrophoresis $(120 \mathrm{~V}, 30 \mathrm{~min})$ to detect the binding ability of PP with siPlk-1. The ability of PP to protect siPlk-1 from the degradation of RNase A was evaluated by the incubation of PP/siPlk-1 nanoparticles with RNase A solution at 37 ${ }^{\circ} \mathrm{C}$ for $30 \mathrm{~min}$ and the further treatment with heparin sodium solution (final concentration: $4 \mathrm{mg} / \mathrm{mL}$ ) at $4{ }^{\circ} \mathrm{C}$ for $60 \mathrm{~min}$. The bands were observed using a Tanon 1600 gel imaging system (Shanghai, China). The morphology of PP/siPlk-1 and PAMAM/siPlk-1 nanoparticles was detected using a HITACHI-H800 transmission electron microscope (TEM). The hydrodynamic diameter and zeta potential of nanoparticles were measured by Nano ZS90 Zetasizer (Malvern Panalytical, UK).

\section{In vitro Transfection Efficiency Assay}

The human HCC cell line HepG2 and hepatocyte L02 were obtained from Shanghai Institute of Cell Bank (Shanghai, China). The HepG2 cells were seeded into 6 -well plates at a density of $1.5 \times 10^{5}$ cells/well and incubated overnight. The nanoparticles were prepared with different carriers and siPlk-1 at various $\mathrm{N} / \mathrm{P}$ ratios, preincubated in $2 \mathrm{~mL}$ FBS-free DMEM for $30 \mathrm{~min}$ and added into corresponding wells. After the incubation for $6 \mathrm{~h}$, the medium was replaced by $10 \%$ FBS-containing DMEM, and the cells were continuously incubated for $48 \mathrm{~h}$, and the fluorescence intensity was measured by CytoFLEX flow cytometer (Beckman Coulter Inc., CA). To evaluate the endosomal escape ability, HepG2 cells were seeded into 6-well plates with sterilized coverslips (initial density: $1.5 \times 10^{5}$ cells/well) and treated by PP/Cy5.5-siPlk-1 (0.5 $\mu \mathrm{g} / \mathrm{mL}$ siPlk-1) for 2,4 and $6 \mathrm{~h}$. Then, the cells were stained by LysoTracker Green for $30 \mathrm{~min}$ and fixed using $4 \%$ paraformaldehyde, and the nuclei were stained with $0.5 \mu \mathrm{g} / \mathrm{mL}$ 4',6-diamidino-2-phenylindole (DAPI) for 10 min. Finally, the coverslips were observed using LSM-710 confocal laser scanning microscope (CLSM, Carl Zeiss Microscopy LLC, Jena, Germany).

\section{Tumor-Targeting Ability Evaluation}

Briefly, the cells were seeded into 6-well plates at a density of $1.5 \times 10^{5}$ cells/well and cultured overnight, and then treated with different samples including NC, free FAMsiPlk-1, PP/FAM-siPlk-1 and PAMAM/FAM-siPlk-1 (N/P ratio of $30,0.5 \mu \mathrm{g} / \mathrm{mL}$ siPlk-1). After the pretreatment with $1 \mathrm{mM}$ PBA for $1 \mathrm{~h}$, the transfection was conducted for $6 \mathrm{~h}$, and the harvested cells were subjected to the detection on a CytoFLEX flow cytometer (Beckman Coulter Inc., CA). For the CLSM analysis, the cells were seeded into 6-well plates with sterilized coverslips (density: $1.5 \times 10^{5}$ cells/well), and treated with different samples containing Cy5.5-siPlk-1. After the transfection for $6 \mathrm{~h}$, the cells were fixed using $75 \%$ ethanol at $4{ }^{\circ} \mathrm{C}$ for $30 \mathrm{~min}$, and the nuclei were stained with $1 \mu \mathrm{g} / \mathrm{mL}$ DAPI for 10 
min. Finally, the coverslips were observed by LSM-710 CLSM (Carl Zeiss Microscopy LLC, Jena, Germany).

\section{Anti-Proliferative Ability Assay}

The anti-proliferative effect induced by PP/siPlk-1 nanoparticles was evaluated by MTT method, colony formation and Live/Dead cell staining assays. In the MTT method, the cells were inoculated in 96-well plates (density: $8.0 \times 10^{3}$ cells/well) and incubated at $37{ }^{\circ} \mathrm{C}$ for $24 \mathrm{~h}$, and then transfected with different nanoparticles in FBS-free DMEM for $6 \mathrm{~h}(0.5 \mu \mathrm{g} / \mathrm{mL}$ siPlk-1). After the incubation in 10\% FBS-containing DMEM for $48 \mathrm{~h}$, MTT solution was added into each well at a final concentration of $0.5 \mathrm{mg} / \mathrm{mL}$, and the plates were incubated at $37{ }^{\circ} \mathrm{C}$ for $4 \mathrm{~h}$. Then, $150 \mu \mathrm{L}$ dimethyl sulfoxide was added to dissolve the formazan crystals, and the optical density at 520 $\mathrm{nm}$ was detected using an HBS-1096A microplate reader (Detie, Nanjing, China) to calculate the cell viability according to the previous reports. ${ }^{34,35}$ For the colony formation assay, the cells after siPlk-1 transfection were plated in 6-well plates at a density of $1.0 \times 10^{4}$ cells/well and incubated at $37{ }^{\circ} \mathrm{C}$ for 7 days. Then, the cells were washed with PBS three times, fixed in $75 \%$ ethanol and stained with $0.2 \%$ crystal violet. Finally, the colonies were photographed by IX73P1F fluorescence microscopy (Olympus, Tokyo, Japan), and the quantitative analysis was performed according to the measurement of optical density at $578 \mathrm{~nm}$. In Live/Dead cell staining assay, the transfected cells were washed with PBS three times and treated with $200 \mu \mathrm{L}$ live/dead cell staining solution containing $2 \mathrm{mM}$ calcein $\mathrm{AM}$ and $4 \mathrm{mM}$ ethidium homodimer, and the images were recorded using IX73P1F fluorescence microscopy (Olympus, Tokyo, Japan).

\section{Cell Apoptosis and Cell Cycle Arrest Assay}

In the cell apoptosis analysis, HepG2 cells were inoculated into 6-well plates at an initial density of $1.5 \times 10^{5}$ cells/ well, and transfected with different nanoparticles (N/P ratio of $30,0.5 \mu \mathrm{g} / \mathrm{mL}$ siPlk-1) in FBS-free DMEM for $6 \mathrm{~h}$. Afterwards the cells were cultured in 10\% FBScontaining DMEM at $37{ }^{\circ} \mathrm{C}$ for $48 \mathrm{~h}$, and stained with Annexin V-FITC and PI according to the apoptosis detection kit's instructions. For the cell cycle arrest analysis, the transfection of siPlk-1 was conducted as described above, and the cells were resuspended in PBS, fixed by $75 \%$ ethanol, and stained with PI at $4{ }^{\circ} \mathrm{C}$ for $30 \mathrm{~min}$. Finally, the cell apoptosis and cell cycle arrest were detected using CytoFLEX flow cytometer (Beckman Coulter Inc., CA). To analyze the mechanism of cell apoptosis, the cells after the transfection of siPlk-1 were washed with PBS three times and stained with $\mathrm{JC}-1$ probe at $37{ }^{\circ} \mathrm{C}$ for $20 \mathrm{~min}$ according to the mitochondrial membrane potential assay kit's instructions. The images were captured by IX73P1F fluorescence microscopy (Olympus, Tokyo, Japan).

\section{Quantitative Real-Time PCR (qPCR)}

The cell culture and siPlk-1 transfection were conducted as described in "Cell apoptosis and cell cycle arrest assay". The total RNA was extracted by TRNzol Universal reagent as recommended by the manufacturer, and then $1 \mu \mathrm{g}$ RNA was used to prepare cDNA through reverse transcription PCR by PrimeScript RT Master Mix. The obtained cDNA was used as a template for PCR amplification using SYBR Premix Ex Taq kit as follows: 1 cycle of $94{ }^{\circ} \mathrm{C}$ for $2 \mathrm{~min}$, followed by 30 cycles of denaturation at $94{ }^{\circ} \mathrm{C}$ for $30 \mathrm{~min}$, annealing at $55{ }^{\circ} \mathrm{C}$ for $30 \mathrm{~s}$ and extension at $72{ }^{\circ} \mathrm{C}$ for 1 min. Finally, the copy numbers of Plk-1 were determined by 7500 Fast Real-Time PCR System (Applied Biosystems Inc.), and the data were analyzed through $2^{-\Delta \Delta C t}$ method. The sequence of primers was used as follows:

$\beta$-actin forward primer: 5'-TCTGGCACCACACCTT CTACAATG-3';

reverse primer: 5'-GGATAGCACAGCCTGGATAGC AA-3';

Plk-1 forward primer: 5'- AGCCTGAGGCCCGATA CTACCTAC-3';

reverse primer: 5'- ATTAGGAGTCCCACACAGGGT CTTC-3'.

\section{Western Blotting Analysis}

The cell culture and siPlk-1 transfection were conducted as described in "Cell apoptosis and cell cycle arrest assay". The harvested cells were subjected to the treatment with RadioImmunoPrecipitation Assay (RIPA) lysis buffer to obtain the samples, and the protein concentration was determined using BCA protein assay kit. Equal amounts of proteins were assayed using 12\% SDSPAGE and then transferred to polyvinylidene fluoride (PVDF) membrane by electroblotting. The membrane was blocked with PBST solution (PBS containing 5\% nonfat milk and $0.1 \%$ Tween-20) at $25^{\circ} \mathrm{C}$ for $1 \mathrm{~h}$, and then incubated with primary antibodies at $4{ }^{\circ} \mathrm{C}$ overnight. After washing with PBST twice, the membrane was treated with HRP-labeled secondary antibody at $25{ }^{\circ} \mathrm{C}$ for 
3 h. Finally, the protein bands were visualized by enhanced chemical luminescence reagent (TransGen Biotech., Beijing, China) on a Tanon-2500 imaging system (Shanghai, China).

\section{Anti-Migration Ability Assay}

The anti-migration effect after siPlk-1 transfection was evaluated by wound healing and Transwell migration assays. In the wound healing assay, HepG2 cells were seeded into 6-well plates at a density of $2.5 \times 10^{5}$ cells/ well and cultured for $24 \mathrm{~h}$. The wound was then generated in each well by scratching using a $200-\mu \mathrm{L}$ pipette tip, after which the cells were treated with different nanoparticles in DMEM for $6 \mathrm{~h}$. The width of wound was measured and photographed using IX73P1F fluorescence microscopy (Olympus, Tokyo, Japan) at different time points. In the Transwell migration assay, HepG2 cells after the transfection with different nanoparticles were inoculated into the upper chamber of 24-well Transwell $\left(2.0 \times 10^{4}\right.$ cells), and the lower chamber was filled with $700 \mu \mathrm{L}$ DMEM containing $10 \%$ FBS. After the culture at $37{ }^{\circ} \mathrm{C}$ for $24 \mathrm{~h}$, the chambers were washed with PBS three times, and the cells on the upper surface of membrane were removed carefully. The cells on the lower surface were fixed using 75\% ethanol at $4{ }^{\circ} \mathrm{C}$ for $20 \mathrm{~min}$ and then stained by $0.2 \%$ crystal violet at $25{ }^{\circ} \mathrm{C}$ for $15 \mathrm{~min}$. Finally, the cells were observed using IX73P1F fluorescence microscope (Olympus, Tokyo, Japan), and the absorbance at $570 \mathrm{~nm}$ was measured by HBS-1096A microplate reader (Detie, Nanjing, China) to quantify the cells transferring across the membrane.

\section{Statistical Analysis}

All data were presented as mean value \pm standard deviation (SD), and the statistical significance of differences between experimental groups and control group was calculated using GraphPad Prism 6 by one-way ANOVA complemented with Student's $t$-test $(* p<0.05 ; * * p<0.01)$.

\section{Results and Discussion}

\section{Synthesis and Characterization of PP/ siPlk-I Nanoparticles}

The modification of PAMAM with PBA was conducted to obtain the derivative $\mathrm{PP}$, and the structure was characterized by ${ }^{1} \mathrm{H}$ NMR and ICP-AES indicating that approximate 7.2 PBA molecules were conjugated on the carrier PAMAM, based on the synthetic procedure in our previous report. ${ }^{35}$
First, gel retardation assay was conducted to evaluate the condensation ability of PP with siPlk-1, in which PP/siPlk1 nanoparticles were prepared through the incubation of these two components at different N/P ratios at $25{ }^{\circ} \mathrm{C}$ for 30 min. As shown in Figure S1, siPlk-1 could be completely blocked in the loading well when N/P ratios were higher than 3.0, indicating that PP could efficiently condense siPlk-1 into stable nanoparticles at N/P ratios of $>3.0$. Then, the protective ability of PP for siPlk-1 against the degradation of RNase A was evaluated at a N/P ratio of 3.0 (Figure $\mathrm{S} 2$ ). Clearly, the band of naked siPlk-1 has been completely degraded after the treatment with RNase A, while obvious band could be observed for PP/siPlk-1 nanoparticles after the digestion with RNase A, in which heparin sodium was used to extract siPlk-1 from nanoparticles. These results demonstrated that the carrier PP could efficiently protect siPlk-1 from the degradation of RNase A, and thus it is potential for improving the stability of siPlk-1 during in vivo application.

Further, hydrodynamic diameter and zeta potential of PAMAM/siRNA and PP/siRNA nanoparticles were measured using Nano ZS90 Zetasizer (Malvern Panalytical, UK). As shown in Table S1, the hydrodynamic diameter values of PAMAM/siRNA and PP/siRNA nanoparticles decreased with the improvement of $\mathrm{N} / \mathrm{P}$ ratios, as the formation of nanoparticles was executed in an electronic interaction manner. Meanwhile, the zeta potential values of these two nanoparticles increased with the improved N/ $P$ ratios owing to the positively charged characteristics of carriers. In addition, both hydrodynamic diameter and zeta potential values were similar for these two nanoparticles. The morphologic analysis of nanoparticles using TEM showed that they were both spherical in shape, and the particle size was ca. $200 \mathrm{~nm}$ at N/P ratio of 30 (Figure $\underline{\mathrm{S} 3}$ ). In a word, the favorable particle size and positive charge of these nanoparticles will be beneficial to achieve the cellular uptake and then execute the function of siPlk-1.

\section{Tumor-Targeting Ability Analysis}

Since the cellular uptake of nanoparticles is crucial for the function of siPlk-1, we monitored the endocytosis of PP/ siPlk-1 nanoparticles in tumor cells. First, the transfection efficiency of PP/siPlk-1 and PAMAM/siPlk-1 nanoparticles at different N/P ratios was evaluated by flow cytometry, in which siPlk-1 was labelled by FAM. As shown in Figure S4, in comparison to free siPlk-1, obvious green fluorescence could be observed for these two nanoparticles, and PP/siPlk-1 could achieve the highest transfection 
efficiency at N/P ratio of 20. However, PAMAM could facilitate the transfection of siPlk-1 with the highest transfection efficiency at N/P ratio of 10 . All these results demonstrated that both these nanoparticles could realize the cellular uptake of siRNA and then execute the function of gene silencing. Then, the tumor-targeting ability of PP/ siPlk-1 nanoparticles was evaluated in HepG2 cells to check the influence of PBA. As shown in Figure 1A, naked siPlk-1 could not enter HepG2 cells, while both PAMAM/siPlk-1 and PP/siPlk-1 exhibited favorable transfection efficiency. Notably, the PBA pretreatment could obviously weaken the transfection of PP/siPlk-1 and had almost no effects on the transfection of PAMAM/siPlk-1. The phenomenon was mainly caused by the shielding of sialic acids by the pretreatment of PBA, which would then influence the interaction of PBA in the derivative PP with sialic acids. Instead, the pretreatment of PBA did not affect the transfection efficiency of PP/siPlk-1 and PAMAM/ siPlk-1 in L02 cells (Figure 1B), owing to the low expression level of sialic acids in L02 cells. ${ }^{31}$ The tumortargeting ability of PP/siPlk-1 was further verified through CLSM, in which Cy5.5-labelled siPlk-1 was used. As shown in Figure S5, PP could successfully mediate the delivery of siPlk-1 into HepG2 cells, which was mainly deposited around the nucleus. After the pretreatment with PBA, red fluorescence of PP/siPlk-1 dramatically decreased. In contrast, the PBA pretreatment did not influence the transfection of PAMAM/siPlk-1. These results were consistent with the flow cytometric analysis, demonstrating that PP could deliver siPlk-1 into tumor cells with favorable specificity and efficiency owing to the recognition of $\mathrm{PBA}$ toward the sialic acid receptors expressed on the surface of tumor cells.

As lysosomal escape is vital for the function of PP/siPlk1 , the intracellular trafficking of PP/siPlk-1 was detected in HepG2 cells in which the co-localization of siPlk-1 and lysosomes were analyzed at different time. As shown in Figure 2, yellow color could be clearly detected in the merged image after the transfection for $4 \mathrm{~h}$ confirming that siPlk-1 was located in lysosomes. When the transfection was extended to $6 \mathrm{~h}$, red and green fluorescence representing siPlk-1 and lysosomes became more distinct, indicating the onset of siPlk-1 escaping from lysosomes. After $8 \mathrm{~h}$, considerable red fluorescence could be observed depleted from the lysosomes, which was caused by the "proton-sponge" effect of PP. Thus, we concluded that PP/siPlk-1 nanoparticles could achieve the lysosomal escape after the transfection for $6 \mathrm{~h}$, which was employed in the subsequent studies for analyzing the function of siPlk-1.

\section{Anti-Proliferative Effects of PP/siPlk-I Nanoparticles}

Inspired by the endocytosis and lysosome escape ability of PP/ siPlk-1, we performed MTT experiment to evaluate the antiproliferative effects of PP/siPlk-1. As shown in Figure 3A, the carrier PP exhibited low cytotoxicity at relatively high concentrations, indicating its favorable biocompatibility. When HepG2 cells were treated with PP/siPlk-1 nanoparticles at N/ $P$ ratio of 30 , the cell survival ratio declined to $66.80 \%$, with $21.52 \%$ inhibition of cell proliferation based on the comparison with the cell viability treated with PP. Simultaneously, the

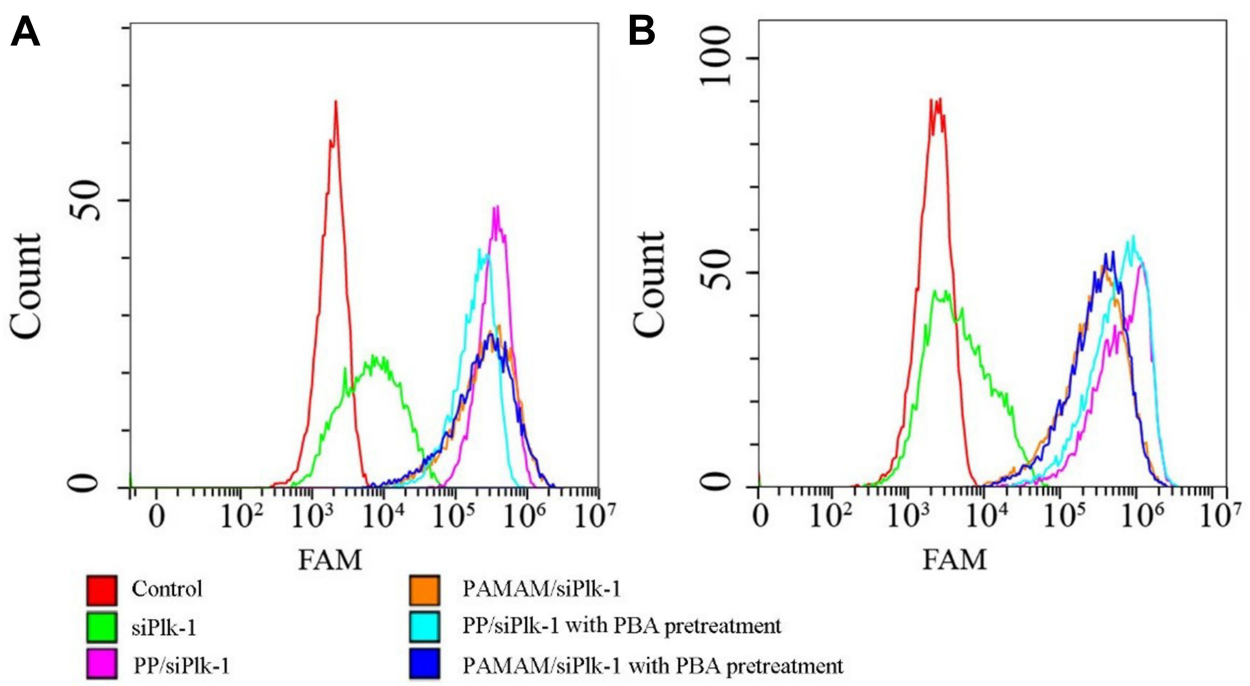

Figure I Tumor-targeting ability of PP/siPlk-I and PAMAM/siPlk-I nanoparticles in HepG2 (A) and L02 cells (B) evaluated by flow cytometry. 


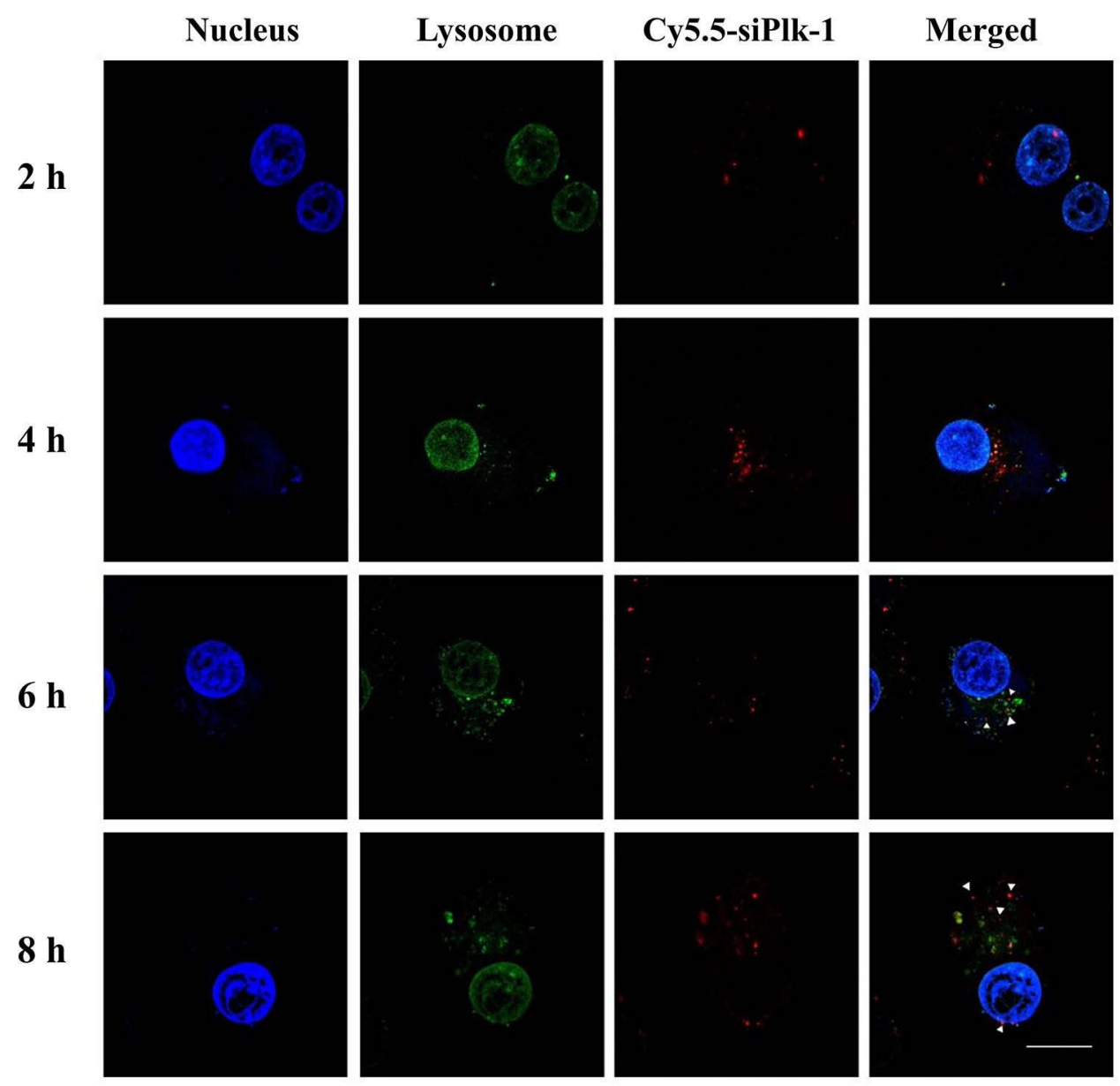

Figure 2 Lysosomal escape analysis of PP/siPlk-I by CLSM. The scale bar is $20 \mu \mathrm{m}$. Blue: DAPI, nucleus; red: Cy5.5-labelled siPlk-I; green: lysosomes, LysoTracker Green.
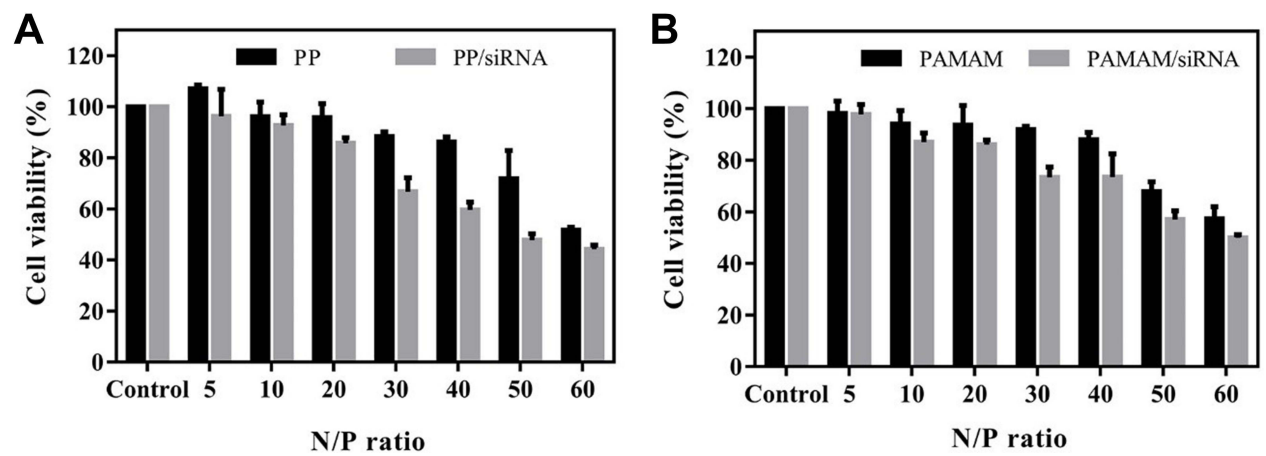

Figure 3 Anti-proliferative effect of PP/siPlk-I (A) and PAMAM/siPlk-I (B) in HepG2 cells detected by the MTT method. Data were presented as mean value \pm SD of triplicate experiments.

viability of HepG2 cells treated with PAMAM/siPlk-1 was measured, which demonstrated that the cell viability decreased gradually with the increasing N/P ratio (Figure 3B). The inhibition of cell proliferation was measured to be $18.48 \%$ for PAMAM/siPlk-1 nanoparticles at N/P ratio of 30. The colony formation assay was performed to provide further insight into the effects of siPlk-1 transfection on the cell proliferation. As shown in Figure S6, the number of colonies decreased obviously after the treatment with PP/siPlk-1, indicating that PP/siPlk-1 could restrain the formation of cell colony, much stronger than PAMAM/siPlk-1. In addition, live/dead cell staining was carried out aiming to illustrate the inhibition of cell proliferation more comprehensively, in which live cells were stained to be green by calcein AM and dead cells emitted 
red fluorescence owing to the function of ethidium homodimer. As shown in Figure S7, almost all the cells were stained to be green in the control group and in the naked siPlk-1 group. In contrast, obvious red fluorescence could be detected for the cells after the transfection with PP/siPlk-1 and PAMAM/siPlk1 nanoparticles, with more dead cells of red color for the PP/ siPlk-1 group. Thus, we concluded that the transfection of siPlk-1 could obviously inhibit the cell proliferation, and the PP-mediated siPlk-1 transfection exhibited stronger antiproliferative effects owing to its favorable tumor-targeting ability and transfection efficiency.

\section{Cell Apoptosis and Cell Cycle Arrest Induced by PP/siPIk-I Nanoparticles}

To illustrate the anti-proliferative mechanism of PP/siPlk-1 and PAMAM/siPlk-1, the apoptosis of HepG2 cells after siPlk-1 transfection was evaluated by flow cytometry based on the staining of Annexin V-FITC and PI. As shown in Figure 4, in comparison to control group, the treatment with free siPlk-1 did not trigger the cell apoptosis (A and D).
Notably, the transfection of siPlk-1 could afford to obvious cell apoptosis, with apoptotic ratios of $27.33 \%$ and $20.32 \%$ using the carriers PP and PAMAM, respectively (E and F). These results were attributed to the efficient delivery of siPlk-1 in tumor cells under the assistance of carriers, and meanwhile PP exhibited higher delivery efficacy of siPlk-1 than PAMAM. In addition, slight cell apoptosis could be detected for the PAMAM/NC and PP/NC groups (B and C), which was probably caused by the intrinsic cytotoxicity of carriers. Also, the weak off-target effect of $\mathrm{NC}$ was another possible reason for the phenomenon, which has been observed in other relevant reports. ${ }^{12,39}$ Further, the cell cycle arrest induced by siPlk-1 delivery was analyzed by flow cytometry after the cell staining with PI. The phase distribution of cells treated by different nanoparticles was shown in Figure 5A, and the values of cell cycle distribution were summarized in Figure 5B. Compared with the control group, there were more cells arrested at G2 phase $(24.69 \%)$ after the treatment with PP/siPlk-1 nanoparticles for 48 h. Meanwhile, the cells treated with PAMAM/siPlk-1

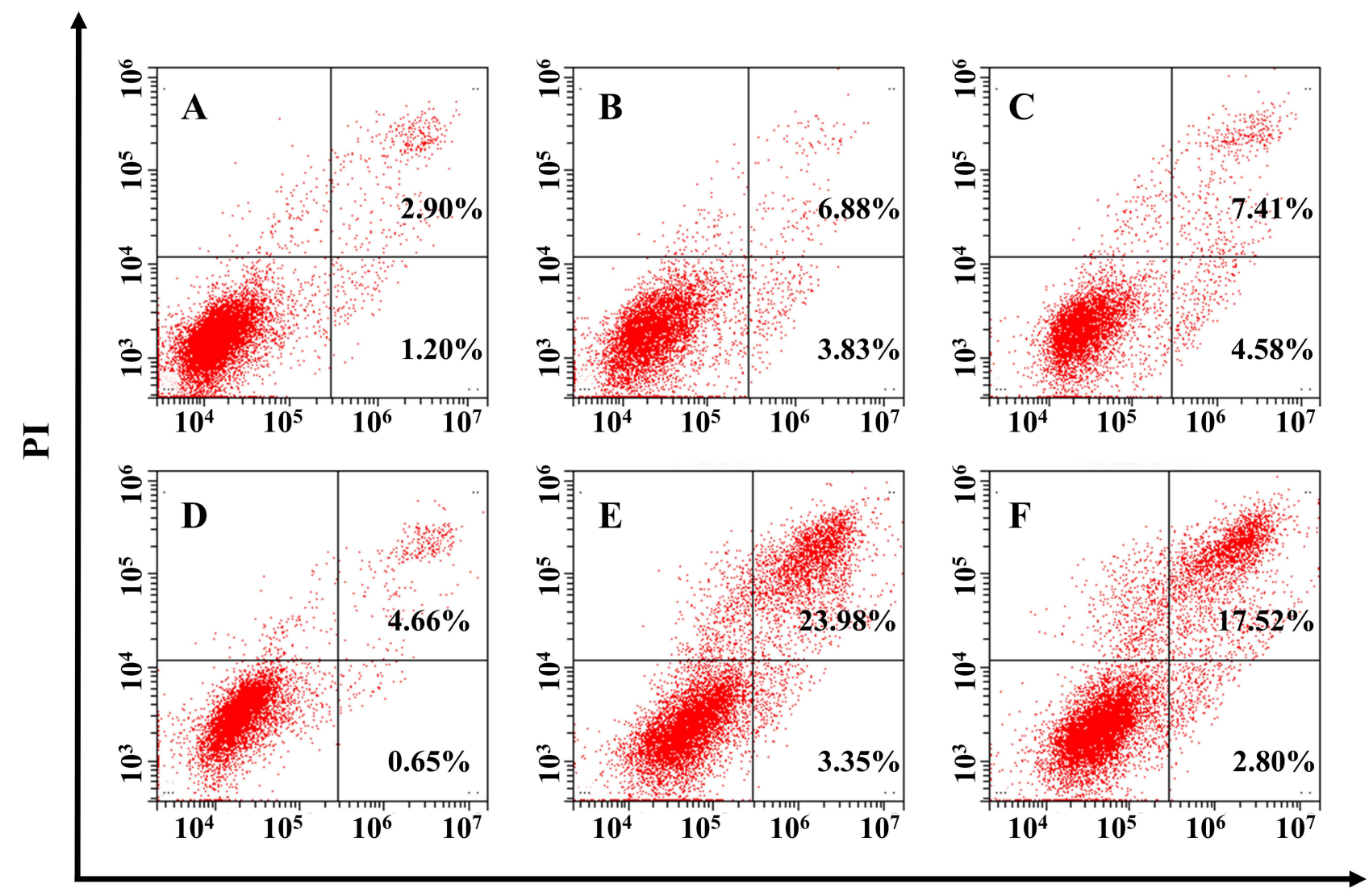

Annexin V-FITC

Figure 4 Flow cytometric analysis of the cell apoptosis after siPlk-I transfection: (A) Control, (B) PP/NC, (C) PAMAM/NC, (D) siPlk-I, (E) PP/siPlk-I and (F) PAMAM/ siPlk-I. 
A
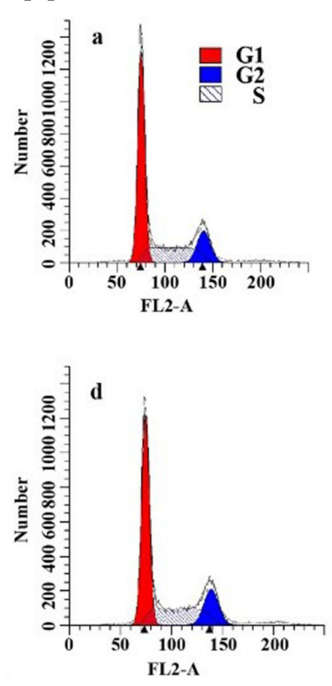
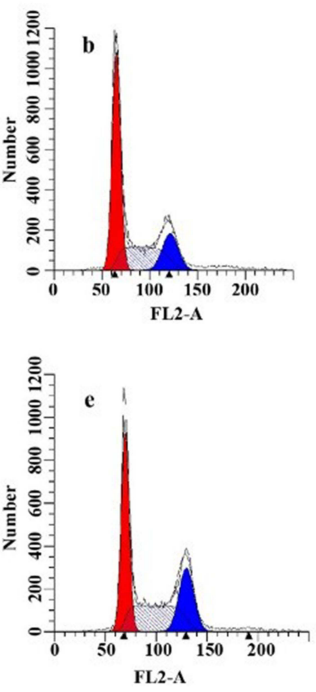

B
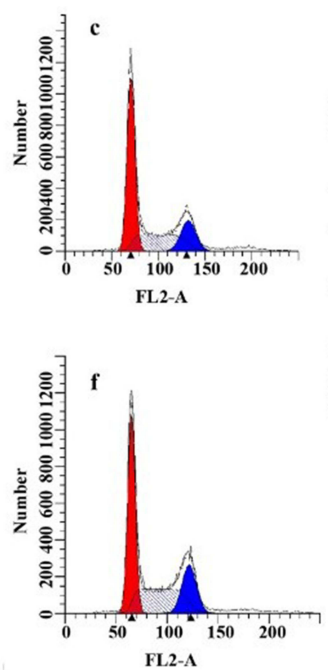

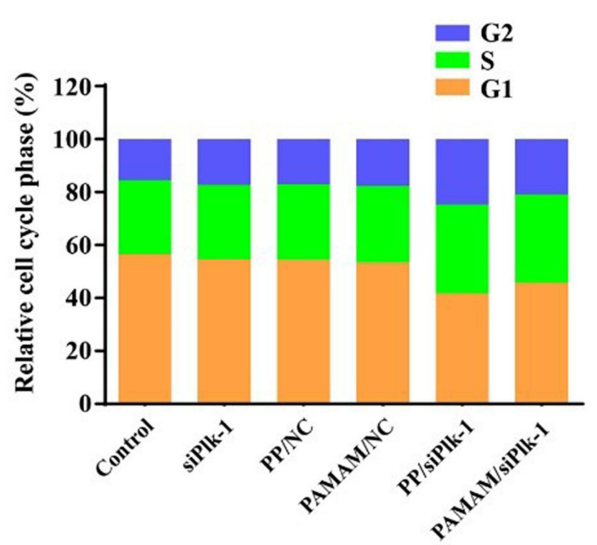

Figure 5 Flow cytometric analysis for the cell cycle arrest of HepG2 cells after siPlk-I transfection (A) and the quantitative analysis (B): (a) control, (b) PP/NC, (c) PAMAM/ NC, (d) siPlk-I, (e) PP/siPlk-I and (f) PAMAM/siPlk-I.

also showed the cell cycle arrest at G2 phase, but the proportion of cells at G2 phase was significantly lower than that of PP/siPlk-1 group. These results were mainly associated with the improved tumor-targeting ability and transfection efficiency of PP. In conclusion, the PPmediated siPlk-1 delivery could contribute to both cell apoptosis and cell cycle arrest at G2 phase, which were key factors in inducing the anti-tumor efficacy.

To investigate the mechanism involved in the inhibition of cell proliferation, qPCR and Western blotting assays were performed to detect the expression levels of important targets after siPlk-1 transfection. As shown in Figure 6, similar expression levels of Plk-1 could be found at mRNA level for the groups of control, siPlk-1, PAMAM/NC and PP/NC, using qPCR analysis. However, the expression level of Plk-1 has been observed to be decreased after the delivery of siPlk-1 using PP and PAMAM as carriers. Meanwhile, PP/siPlk-1 could suppress the Plk-1 expression more obviously than PAMAM/siPlk-1 group, with $44.97 \%$ relative expression level. Similarly, the reduced Plk-1 expression could be observed at the protein level after siPlk-1 transfection (Figure S8), which was in accordance with the qPCR results. Additionally, decreased procaspase 3 level was detected after the delivery of siPlk-1, which meant the activation of caspase 3 and the occurrence of apoptotic effect. As the activation of caspase 3 could catalyze the hydrolysis of many protein substrates, ${ }^{40}$ the expression level of PARP could be clearly observed to be decreased after the siPlk-1 delivery. The expression of procaspase 8 and 9 simultaneously decreased which

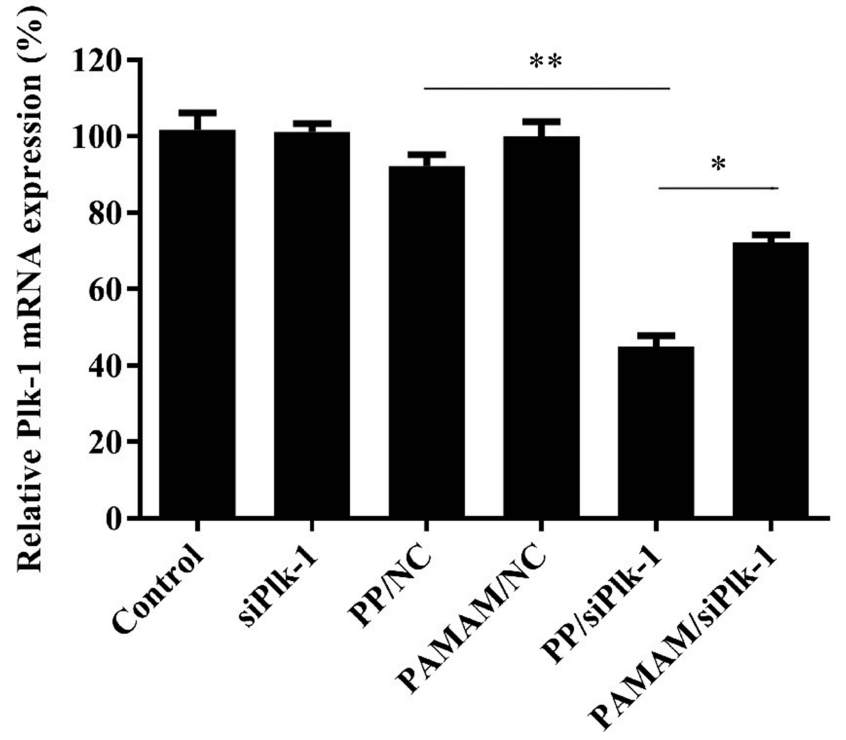

Figure 6 Evaluation of Plk-I expression at mRNA level by qPCR. Data were presented as mean value \pm SD of triplicate experiments $\left(* p<0.05\right.$, $\left.*^{*} p<0.01\right)$.

meant the cleavage of precursor proteins to activate caspase 8 and 9, suggesting that the carriers-mediated the delivery of siPlk-1 induce the cell apoptosis via both the death receptorand mitochondria-dependent signaling pathways. Enhanced expression of p53 and decreased Bcl-2 level could also be found following the treatment with $\mathrm{PP} / \mathrm{siPlk}-1$ nanoparticles, both of which contributed to the apoptosis of tumor cells.

Since the mitochondrial membrane potential depolarizes during the early stage of cell apoptosis, ${ }^{40}$ the changes in mitochondrial membrane potential of HepG2 cells were 
monitored following the treatment with different nanoparticles, using JC-1 dye as an indicator (Figure S9). When the depolarization of mitochondrial membrane potential occurs, the fluorescence will change from red to green, owing to the conversion of JC-1 from aggregates to monomeric state. Compared with PAMAM/siPlk-1 group, more cells with green fluorescence could be detected after the transfection of PP/siPlk-1, implying the higher apoptosis-inducing ability of PP/siPlk-1 nanoparticles. In addition, a small number of cells with green fluorescence could be clearly visualized after the PP/NC and PAMAM/NC treatment, which was probably caused by the slight cytotoxicity of these two carriers.

\section{Inhibition of Cell Migration and Invasion by PP/siPlk-I Nanoparticles}

The migration and invasion ability of tumor cells are closely related to tumor metastasis. ${ }^{41}$ Thus, the effects of $\mathrm{PP} /$ siPlk-1 delivery on the migration and invasion were evaluated using wound healing and Transwell migration assays. As shown in Figure 7, there were no obvious differences in the cells which have migrated to the lower chamber of Transwell between the control and siPlk-1 groups, due to the insufficient transfection of siPlk-1. The PP- or PAMAM-mediated the delivery of siPlk-1 could dramatically reduce the migrating cell number, which indicated the successful inhibition of cell migration and invasion. Meanwhile, PP/siPlk-1 nanoparticles exhibited stronger inhibition of cell migration and invasion than PAMAM/siPlk-1 group. It was noted that the NC transfection could slightly suppress the cell migration rate, which was similar to the effect of $\mathrm{NC}$ on cell apoptosis. Moreover, wound healing assay showed that siPlk-1 delivery using PP or PAMAM as carriers could dramatically hinder the wound healing, in which higher anti-migration effect was achieved for the PP/siPlk-1 group (Figure S10). These results were consistent with Transwell migration
A
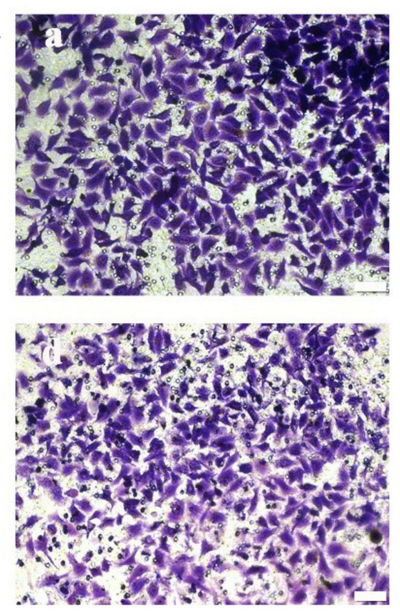
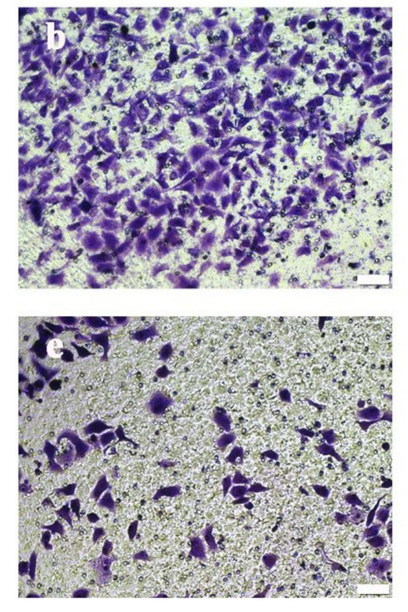
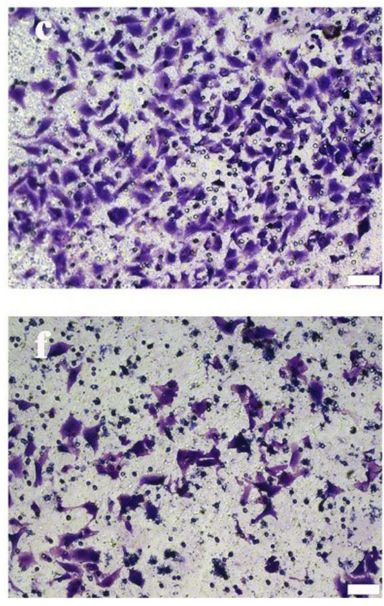

B

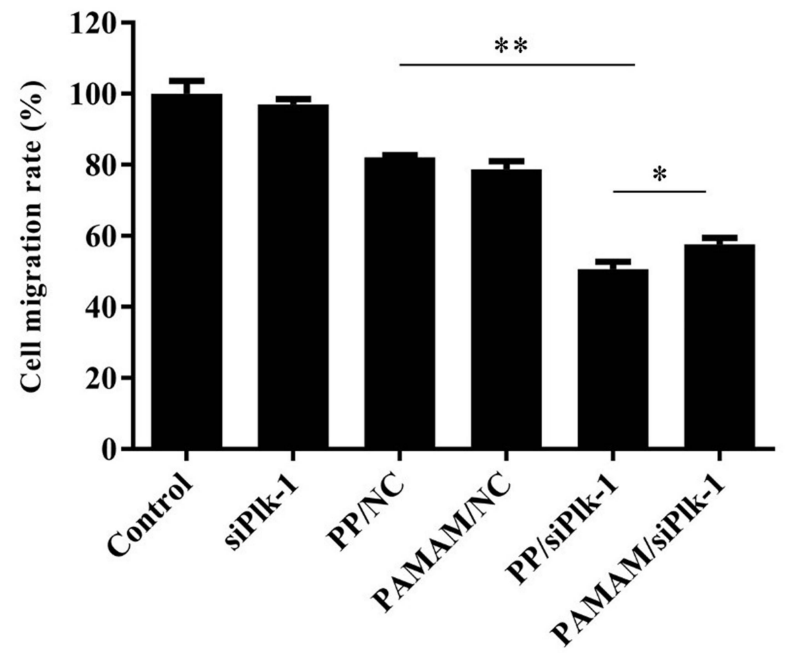

Figure 7 Transwell migration assay of HepG2 cells after the siPlk-I transfection (A) and the quantitative analysis (B): (a) control, (b) PP/NC, (c) PAMAM/NC, (d) siPlk-I, (e) PP/siPlk-I and (f) PAMAM/siPlk-I. The scale bar was $50 \mu \mathrm{m}$. Data were presented as mean value \pm SD of triplicate experiments $\left({ }^{*} p<0.05,{ }^{* *} p<0.01\right)$. 
assay, which illustrated that PP/siPlk-1 delivery exerted significant inhibition on the migration and invasion of tumor cells. Thus, it is beneficial to solve the tumor recurrence based on the induction of anti-proliferation and antimigration effects, which has been considered as an important factor in the high lethality of patients with tumors.

\section{Conclusion}

In conclusion, a tumor-targeting dendrimer PP has been successfully developed through the introduction of PBA on the carrier PAMAM, and then used as a delivery system to achieve the transfection of siPlk-1 in tumor cells. Based on the favorable tumor-targeting ability and transfection efficiency of PP, siPlk-1 delivery could efficiently suppress the expression level of Plk-1 at mRNA and protein levels, which was accompanied by a distinct inhibition of cell proliferation and migration as well as the apoptosis and cell cycle arrest of tumor cells. Overall, the derivative PP has been demonstrated to be an ideal tumor-targeting carrier for the delivery of oligonucleotides and therapeutic genes, which shows great potential in the gene therapy of malignant tumors and other genetic diseases.

\section{Acknowledgments}

The work was supported by National Key R\&D Program of China (2020YFA0907003), National Natural Science Foundation of China (32071267 and 81872928), Science and Technology Department of Jilin Province (20190201288JC), Development and Reform Commission of Jilin Province (2021C041-4) and Interdisciplinary Innovation Program of Jilin University (JLUXKJC2020308).

\section{Disclosure}

The authors report no conflicts of interest in this work.

\section{References}

1. Miller KD, Nogueira L, Mariotto AB, et al. Cancer treatment and survivorship statistics, 2019. CA Cancer J Clin. 2019;69:363-385. doi:10.3322/caac. 21565

2. Duan F, Lam MG. Delivery approaches of gene therapy in hepatocellular carcinoma. Anticancer Res. 2013;33:4711-4718.

3. Grandhi MS, Kim AK, Ronnekleiv-Kelly SM, et al. Hepatocellular carcinoma: from diagnosis to treatment. Surg Oncol. 2016;25:74-85. doi:10.1016/j.suronc.2016.03.002

4. Zucman-Rossi J, Villanueva A, Nault JC. Genetic landscape and biomarkers of hepatocellular carcinoma. Gastroenterology. 2015;149:1226-1239. doi:10.1053/j.gastro.2015.05.061

5. Sun W, Shi Q, Zhang H, et al. Advances in the techniques and methodologies of cancer gene therapy. Discov Med. 2019;27:45-55.
6. Buduru S, Zimta AA, Ciocan C, et al. RNA interference: new mechanistic and biochemical insights with application in oral cancer therapy. Int J Nanomed. 2018;13:3397-3409. doi:10.2147/IJN.S167 383

7. Mahmoodi CG, Dana H, Gharagouzloo E, et al. Small interfering RNAs (siRNAs) in cancer therapy: a nano-based approach. Int J Nanomed. 2019;14:3111-3128. doi:10.2147/IJN.S200253

8. Sullivan KM, Kenerson HL, Pillarisetty VG, et al. Precision oncology in liver cancer. Ann Transl Med. 2018;6:285. doi:10.21037/ atm.2018.06.14

9. Macurek L, Lindqvist A, Lim D, et al. Polo-like kinase-1 is activated by Aurora A to promote checkpoint recovery. Nature. 2008;455:119-123. doi:10.1038/nature07185

10. Hirai Y, Saeki R, Song F, et al. Charge-reversible lipid derivative: a novel type of $\mathrm{pH}$-responsive lipid for nanoparticle-mediated siRNA delivery. Int $J$ Pharm. 2020;585:119479. doi:10.1016/j.ijpharm.20 20.119479

11. Ji M, Li P, Sheng N, et al. Sialic acid-targeted nanovectors with phenylboronic acid-grafted polyethylenimine robustly enhance siRNA-based cancer therapy. ACS Appl Mater Interfaces. 2016;8:9565-9576. doi:10.1021/acsami.5b11866

12. Hou L, Song Z, Xu Z, et al. Folate-mediated targeted delivery of siPLK1 by leucine-bearing polyethylenimine. Int $J$ Nanomed. 2020;15:1397-1408. doi:10.2147/IJN.S227289

13. Zheng M, Liu Y, Wang Y, et al. ROS-responsive polymeric siRNA nanomedicine stabilized by triple interactions for the robust glioblastoma combinational RNAi therapy. Adv Mater. 2019;31:e1903277. doi:10.1002/adma.201903277

14. Pack DW, Hoffman AS, Pun S, et al. Design and development of polymers for gene delivery. Nat Rev Drug Discov. 2005;4:581-593. doi:10.1038/nrd1775

15. Foldvari M, Chen DW, Nafissi N, et al. Non-viral gene therapy: gains and challenges of non-invasive administration methods. J Control Release. 2016;240:165-190. doi:10.1016/j.jconrel.2015.12.012

16. Kawata E, Ashihara E, Kimura S, et al. Administration of PLK-1 small interfacing RNA with atelocollagen prevents the growth of live metastases of lung cancer. Mol Cancer Ther. 2008;7:2904-2912. doi:10.1158/1535-7163.MCT-08-0473

17. Deng H, Jiang Q, Yang Y, et al. Intravenous liposomal delivery of the short hairpin RNAs against Plk1 controls the growth of established human hepatocellular carcinoma. Cancer Biol Ther. 2011;11: 401-409. doi:10.4161/cbt.11.4.14178

18. Xiang B, Dong D, Shi N, et al. PSA-responsive and PSMA-mediated multifunctional liposomes for targeted therapy of prostate cancer. Biomaterials. 2013;34:6976-6991. doi:10.1016/j.biomaterials.2013. 05.055

19. Wang Y, Li J, Chen Y, et al. Balancing polymer hydrophobicity for ligand presentation and siRNA delivery in dual function CXCR4 inhibiting polyplexes. Biomater Sci. 2015;3:1114-1123. doi:10. 1039/C5BM00003C

20. Greco KA, Franzen CA, Foreman KE, et al. PLK-1 silencing in bladder cancer by siRNA delivered with exosomes. Urology. 2016;91:e1-7. doi:10.1016/j.urology.2016.01.028

21. Alex MRA, Veeranarayanan S, Poulose AC, et al. Click modified amphiphilic graft copolymeric micelles of poly(stryrene-alt-maleic anhydride) for combinatorial delivery of doxorubicin and plk-1siRNA in cancer therapy. J Mater Chem B. 2016;4:7303-7313. doi:10.1039/C6TB02094A

22. Alex MRA, Nehate C, Veeranarayanan S, et al. Self assembled dual responsive micelles stabilized with protein for co-delivery of drug and siRNA in cancer therapy. Biomaterials. 2017;133:94-106. doi:10.1016/j.biomaterials.2017.04.022

23. Ewe A, Noske S, Karimov M, et al. Polymeric nanoparticles based on tyrosine-modified, low molecular weight polyethylenimines for siRNA delivery. Pharmaceutics. 2019;11:600. doi:10.3390/ pharmaceutics 11110600 
24. Venditto VJ, Regino CA, Brechbiel MW, et al. PAMAM dendrimer based macromolecules as improved contrast agents. Mol Pharm. 2005;2:302-311. doi:10.1021/mp050019e

25. Li L, Wei Y, Gong C. Polymeric nanocarriers for non-viral gene delivery. J Biomed Nanotechnol. 2015;11:739-770. doi:10.1166/jbn.2015.2069

26. Li J, Liang H, Liu J, et al. Poly(amidoamine) (PAMAM) dendrimer mediated delivery of drug and pDNA/siRNA for cancer therapy. Int J Pharm. 2018;546:215-225. doi:10.1016/j.ijpharm.2018.05.045

27. Chen W, Liu Y, Liang X, et al. Chondroitin sulfate-functionalized polyamidoamine as a tumor-targeted carrier for miR-34a delivery. Acta Biomater. 2017;57:238-250. doi:10.1016/j.actbio.2017.05.030

28. Wang M, Cheng Y. Structure-activity relationships of fluorinated dendrimers in DNA and siRNA delivery. Acta Biomater. 2016;46:204-210. doi:10.1016/j.actbio.2016.09.023

29. Han H, Yang J, Wang Y, et al. Nucleobase-modified polyamidoamine-mediated miR-23b delivery to inhibit the proliferation and migration of lung cancer. Biomater Sci. 2017;5:2268-2275. doi:10.1039/C7BM00599G

30. Chang H, Zhang Y, Li L, et al. Efficient delivery of small interfering RNA into cancer cells using dodecylated dendrimers. $J$ Mater Chem B. 2015;3:8197-8202. doi:10.1039/C5TB01257K

31. Wu D, Yang J, Xing Z, et al. Phenylboronic acid-functionalized polyamidoamine-mediated Bcl-2 siRNA delivery for inhibiting the cell proliferation. Colloids Surf B Biointerfaces. 2016;146:318-325. doi:10.1016/j.colsurfb.2016.06.034

32. Han H, Chen W, Yang J, et al. 2-Amino-6-chloropurine-modified polyamidoamine-mediated p53 gene transfection to achieve anti-tumor efficacy. New J Chem. 2018;42:13375-13381. doi:10. 1039/C8NJ01870G

33. Yang J, Zhang J, Liu Y, et al. Phenylboronic acid-modified polyamidoamine-mediated delivery of short GC rich DNA for hepatocarcinoma gene therapy. Biomater Sci. 2019;7:3348-3358. doi:10. 1039/C9BM00394K
34. Song Z, Liang X, Wang Y, et al. Phenylboronic acid-functionalized polyamidoamine-mediated miR-34a delivery for the treatment of gastric cancer. Biomater Sci. 2019;7:1632-1642. doi:10.1039/ C8BM01385C

35. Yang J, Zhang J, Xing $J$, et al. Inhibition of proliferation and migration of tumor cells through phenylboronic acid-functionalized polyamidoamine-mediated delivery of a therapeutic DNAzyme Dz13. Int J Nanomed. 2019;14:63 71-6385. doi:10.2147/IJN.S211744

36. Matsumoto A, Cabral H, Sato N, et al. Assessment of tumor metastasis by the direct determination of cell-membrane sialic acid expression. Angew Chem Int Ed. 2010;49:5494-5497. doi:10.1002/ anie. 201001220

37. Yin J, Hashimoto A, Izawa M, et al. Hypoxic culture induces expression of sialin, a sialic acid transporter, and cancer-associated gangliosides containing non-human sialic acid on human cancer cells. Cancer Res. 2006;66:2937-2945. doi:10.1158/0008-5472.CAN-052615

38. Deshayes S, Cabral H, Ishii T, et al. Phenylboronic acid-installed polymeric micelles for targeting sialylated epitopes in solid tumors. J Am Chem Soc. 2013;135:15501-15507. doi:10.1021/ ja406406h

39. Xing Z, Gao S, Duan Y, et al. Delivery of DNAzyme targeting Aurora kinase A to inhibit the proliferation and migration of human prostate cancer. Int J Nanomed. 2015;10:5715-5727.

40. Zhang J, Wu D, Xing Z, et al. N-Isopropylacrylamide-modified polyethylenimine-mediated p53 gene delivery to prevent the proliferation of cancer cells. Colloids Surf $B$ Biointerfaces. 2015;129:54-62. doi:10.1016/j.colsurfb.2015.03.032

41. Su Y, Li X, Ji W, et al. Small molecule with big role: microRNAs in cancer metastatic microenvironments. Cancer Lett. 2014;344: 147-156. doi:10.1016/j.canlet.2013.10.024
International Journal of Nanomedicine

\section{Publish your work in this journal}

The International Journal of Nanomedicine is an international, peerreviewed journal focusing on the application of nanotechnology in diagnostics, therapeutics, and drug delivery systems throughout the biomedical field. This journal is indexed on PubMed Central, MedLine, CAS, SciSearch ${ }^{\mathbb{B}}$, Current Contents ${ }^{\mathbb{B}} /$ Clinical Medicine, $^{2}$

\section{Dovepress}

Journal Citation Reports/Science Edition, EMBase, Scopus and the Elsevier Bibliographic databases. The manuscript management system is completely online and includes a very quick and fair peer-review system, which is all easy to use. Visit http://www.dovepress.com/ testimonials.php to read real quotes from published authors. 